\title{
Mathematical formulation for estimation of baseline in synthetic aperture radar interferometry
}

\author{
R K GOYAL and A K VERMA \\ Defence Electronics Applications Laboratory, Post Box No. 54, Raipur Road, \\ Dehradun 248 001, India
}

MS received 29 May 1995; revised 5 October 1995

\begin{abstract}
Terrain height estimation through spaceborne interferometric synthetic aperture radar (INSAR) requires accurate knowledge of the orbital shift between repeat passes. Mathematical models are available for the estimation of horizontal orbital shift. However, in reality, the orbital shift between repeat passes is modelled as two-dimensional for the same azimuth scanline. In this paper, a new mathematical formulation has been developed for the estimation of the two-dimensional orbital shift of INSAR based on the fringe line pattern in the interferogram of flat earth.
\end{abstract}

Keywords. Interferometric synthetic aperture radar; baseline estimation; horizontal orbital shift; fringe line pattern.

\section{Introduction}

Topographic maps of the terrain surface have found numerous uses in the areas of geology, natural resources, land use management, hydrology, remote sensing etc. In general, topographic maps can be generated by using stereo pair optical photographs or stereo pair radar imagery in both of which the resolution depends on the ground cell size. In both airborne or spaceborne Interferometric Synthetic Aperture Radar (INSAR), two SAR phase imageries of the same scene are combined coherently to form a phase interferogram which can be used to derive the information of the terrain elevation (Zebker \& Goldstein 1986). In the airborne INSAR system, two antennae are used simultaneously to receive the signals, whereas, in the repeat pass orbit INSAR, a single antenna is used in the satellite or spaceborne system and two images are taken for the same scene during different orbital passes and then combined (Zebker \& Goldstein 1986; Madsen et al 1993). The phase difference between the two passes contains information about the ground elevation, which varies from 0 to $2 \pi$ due to rotation of the phase vector. The performance of the radar interferometer depends upon system parameters such as frequency, resolution, orbital parameters (baseline vector) and errors introduced during data processing and post-processing operations due 
to signal-to-noise ratio, number of looks, pixel misregistration, baseline decorrelation and phase aliasing (Li \& Goldstein 1990; Lin et al 1991, 1992; Hagberg \& Ulander 1993). In repeat pass interferometry, significant error results from inaccuracies in the knowledge of INSAR orbital shift (also called the baseline), used in the mathematical models available (Zebker \& Goldstein 1986; Lin et al 1991) to derive a topographic map. For each pixel corresponding to a given point of the area in both images, the phase difference value gives the measure of difference in path length from a given pixel to each antenna of the SAR interferometer. Using the knowledge of orbital parameters and the phase difference interferogram, digital terrain elevation can be directly related to the altitude on a pixel-by-pixel basis.

For a flat earth, the phase difference value at a point increases from zero to $2 \pi$, then drops to zero again, forming a saw-tooth pattern in the range direction (Lin et al 1992). The average fluctuations in the adjacent pixels are very small. The sharp transition of phase difference value from $2 \pi$ to zero is called a fringe line in the phase interferogram (Lin et al 1991). The two complex images are combined to form a phase difference image called an interferogram. For a flat earth, many fringe lines of a definite pattern in the interferogram give precise information of baseline vectors or orbital parameters during repeat passes (Lin et al 1991, 1992). Before applying the mathematical models, the phase interferogram image is unwrapped by adding $2 \pi$ wherever fringe lines occur. For the two images to be properly registered $\Delta y$ shift of the satellite repeat pass orbit is zero and $\Delta x, \Delta z$ shifts are present to form the baseline vector. The variation in the fringe pattern contains the information about surface topography. However, in the presence of phase noise, it becomes difficult to decide where the transition of phase difference value from $2 \pi$ to 0 occurs. Different phase-unwrapping techniques are used to reduce phase noise prior to the estimation of terrain elevation (Lin et al 1992; Madsen et al 1993). For any terrain, the phase difference value varies randomly between the fringe lines. Lin et al (1991) have derived a mathematical formulation to estimate the one-dimensional horizontal shift by knowing the distance between fringe lines. In reality, however, this orbital shift is two-dimensional for the same azimuth scanline. In this paper, a new mathematical formulation is presented based on the detection of three consecutive fringe lines on flat earth to accurately estimate the two-dimensional orbital shift of INSAR. It is observed through modelling that the consecutive fringe lines are formed at such a small distance that the ellipticity of the earth has no bearing on the results and that, in the absence of any topography, the earth can be assumed to be flat between these consecutive fringe lines.

\section{Mathematical formulation}

In INSAR, the complex images (Lin et al 1992) of the same region or area are taken from two orbits successively. A and B are the positions of the satellite in two successive orbits. $\Delta x$ and $\Delta z$ are the horizontal and vertical shifts of position B with respect to position A as shown in figure 1 . The satellite-borne synthetic aperture radar (SAR) operates $h$ metres above the ground and it looks to the side with an incidence angle of $\alpha$ degrees. $P$ and $Q$ are the points of interest which are $z_{1}$ and $z_{2}$ metres above the ground reference. $\Delta h$ is the 


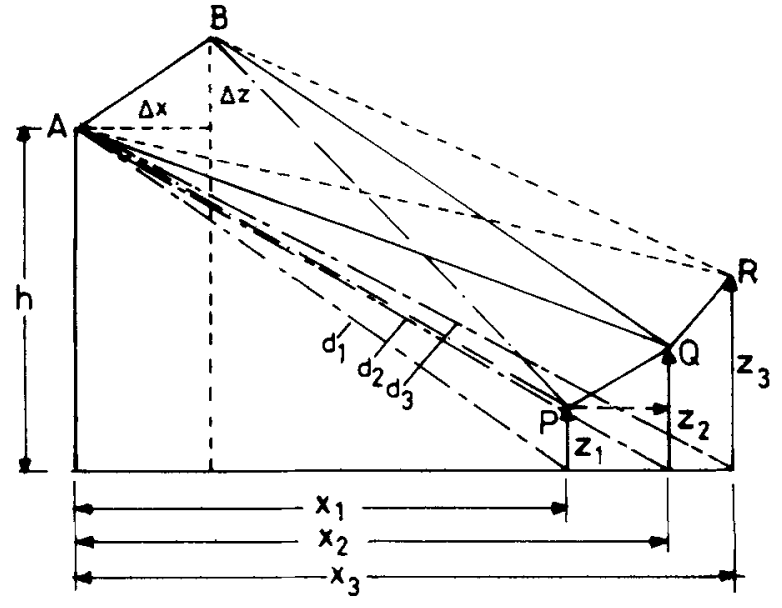

Figure 1. Geometry of interferometric SAR.

relative height between $\mathrm{P}$ and $\mathrm{Q}$. A ground point $\mathrm{P}, x_{1}$ metres away from the nadir, is at a distance $\rho_{1}$ from the first orbit position A and $\rho_{2}$ from the second orbit. Hence, the path length difference at ground position $P$ can be written as

$$
\rho=\rho_{1}-\rho_{2}=\mathrm{AP}-\mathrm{BP} .
$$

Similarly, the path difference $\rho^{\prime}$ at ground position $\mathrm{Q}$ can be written as

$$
\rho^{\prime}=\mathrm{AQ}-\mathrm{BQ} \text {. }
$$

Hence,

$$
\begin{aligned}
\rho= & \mathrm{AP}-\mathrm{BP} \\
= & \left\{\left(h-z_{1}\right)^{2}+x_{1}^{2}\right\}^{1 / 2}-\left\{\left(h-z_{1}+\Delta z\right)^{2}+\left(x_{1}-\Delta x\right)^{2}\right\}^{1 / 2} \\
= & \left\{h^{2}+z_{1}^{2}-2 h z_{1}+x_{1}^{2}\right\}^{1 / 2} \\
& -\left\{h^{2}+z_{1}^{2}-2 h z_{1}+\Delta z^{2}+2 h \Delta z-2 z_{1} \Delta z+x_{1}^{2}+\Delta x^{2}-2 x_{1} \Delta x\right\}^{1 / 2} .
\end{aligned}
$$

As shown in figure $1: h^{2}+x_{1}^{2}=d_{1}^{2}$.

Hence, (3) can be reduced to

$$
\begin{aligned}
\rho= & d_{1}\left\{\left(1+\frac{z_{1}^{2}}{d_{1}^{2}}-\frac{2 h z_{1}}{d_{1}^{2}}\right)^{1 / 2}\right. \\
& \left.-\left(1+\frac{z_{1}^{2}}{d_{1}^{2}}-\frac{2 h z_{1}}{d_{1}^{2}}+\frac{\Delta z^{2}}{d_{1}^{2}}+\frac{2 h \Delta z}{d_{1}^{2}}-\frac{2 z_{1} \Delta z}{d_{1}^{2}}+\frac{\Delta x^{2}}{d_{1}^{2}}-\frac{2 x_{1} \Delta x}{d_{1}^{2}}\right)^{1 / 2}\right\} .
\end{aligned}
$$

Let us assume,

$$
u_{1}=\frac{z_{1}^{2}}{d_{1}^{2}}-\frac{2 h z_{1}}{d_{1}^{2}}
$$


and

$$
u_{2}=\frac{2}{d_{1}^{2}}\left\{h \Delta z-z_{1} \Delta z-x_{1} \Delta x\right\}
$$

Since

$$
\Delta z^{2} / d_{1}^{2} \ll 1 \text { and } \Delta x^{2} / d_{1}^{2} \ll 1,
$$

substituting the value of $u_{1}$ and $u_{2}$, (4) reduces to

$$
\rho=d_{1}\left\{\left(1+u_{1}\right)^{1 / 2}-\left(1+u_{1}+u_{2}\right)^{1 / 2}\right\} .
$$

Using the approximation $(1+u)^{1 / 2}=1+u / 2-u^{2} / 8$, where $u<1$, (5) reduces to

$$
\rho=d_{1}\left\{-\frac{u_{2}}{2}+\frac{u_{2}^{2}}{8}+\frac{u_{1} u_{2}}{4}\right\} .
$$

Since $u_{2}^{2} / 8 \ll 1$, (6) becomes

$$
\rho=\frac{d_{1} u_{2}}{2}\left(-1+\frac{u_{1}}{2}\right) \text {. }
$$

Substituting the values of $u_{1}$ and $u_{2},(7)$ reduces to

$$
\begin{aligned}
\rho= & -\frac{\left(h \Delta z-z_{1} \Delta z-x_{1} \Delta x\right)}{d_{1}} \\
& +\frac{1}{d_{1}^{3}}\left[\frac{h \Delta z z_{1}^{2}}{2}-h^{2} z_{1} \Delta z-\frac{z_{1}^{3} \Delta z}{2}+h z_{1}^{2} \Delta z-\frac{x_{1} \Delta x z_{1}}{2}+x_{1} h z_{1} \Delta x\right] .
\end{aligned}
$$

Since,

$$
\frac{h z_{1}^{2} \Delta z}{2 d_{1}^{3}} \ll 1, \quad \frac{z_{1}^{3} \Delta z}{z d_{1}^{3}} \ll 1, \quad \frac{h z_{1}^{2} \Delta z}{d_{1}^{3}} \ll 1 \quad \text { and } \quad \frac{x_{1} z_{1}^{2} \Delta x}{2 d_{1}^{3}} \ll 1 .
$$

Hence, neglecting these terms, (8) reduces to

$$
\rho=\Delta x\left[\frac{x_{1}}{d_{1}}+\frac{h x_{1} z_{1}}{d_{1}^{3}}\right]-\Delta z\left[\frac{h-z_{1}}{d_{1}}+\frac{h^{2} z_{1}}{d_{1}^{3}}\right] .
$$

Equation (9) estimates the path difference $(\rho)$ for a point $P$ on the ground reference assuming $z_{1} \ll h$ and $x_{1} \gg \Delta x, \Delta z$ shift of satellite position. In the case of the formulation by Lin et al $(1991,1992)$ the perpendicular baseline between two parallel orbits $\Delta x=B_{1}$ is taken assuming $\Delta z=0$. Applying this concept of Lin et al $(1991,1992),(9)$ reduces to Lin's formula and can be written as

$$
\rho=\frac{x_{1} \mathrm{~B}_{1}}{d_{1}}+\frac{x_{1} h z_{1} \mathrm{~B}_{1}}{d_{1}^{3}} .
$$


Similarly, taking only vertical shift $\Delta z=\mathrm{B}_{2}$ and $\Delta x=0$,

$$
\rho=-\frac{\left(h-z_{1}\right) \mathrm{B}_{2}}{d_{1}}-\frac{h^{2} z_{1} \mathrm{~B}_{2}}{d_{1}^{3}}
$$

Equation (9) is of a general form for evaluation of path difference at a point for any combination of satellite shifts (horizontal or vertical) during the repeat pass orbit.

Similarly, the path difference at a point $\mathrm{Q}$ on the ground reference can be derived and written as

$$
\rho^{\prime}=\Delta x\left[\frac{x_{2}}{d_{2}}+\frac{h x_{2} z_{2}}{d_{2}^{3}}\right]-\Delta z\left[\frac{\left(h-z_{2}\right)}{d_{2}}+\frac{h^{2} z_{2}}{d_{2}^{3}}\right] .
$$

The expression for path difference $\rho$ and $\rho^{\prime}$ can also be written as

$$
\begin{aligned}
& \rho=\frac{1}{d_{1}}\left(x_{1} \Delta x-h \Delta z\right)+\frac{z_{1}}{d_{1}}\left(\Delta z+\frac{h x_{1} \Delta x}{d_{1}^{2}}-\frac{h^{2} \Delta z}{d_{1}^{2}}\right), \\
& \rho^{\prime}=\frac{1}{d_{2}}\left(x_{2} \Delta x-h \Delta z\right)+\frac{z_{2}}{d_{2}}\left(\Delta z+\frac{h x_{2} \Delta x}{d_{2}^{2}}-\frac{h^{2} \Delta z}{d_{2}^{2}}\right) .
\end{aligned}
$$

In (9), the first term is independent of $z$ and corresponds to path difference for flat earth. The path difference is proportional to $\Delta x$ and $\Delta z$, and is a nonlinear function of $x_{1}$ or $x_{2}$, since $d_{1}$ or $d_{2}$ is a nonlinear function of $x$. The second term in (9) is due to terrain elevation $z_{1}$ or $z_{2}$. The phase associated with the path difference between any two points $\mathrm{P}\left(x_{1}, z_{1}\right)$ and $\mathrm{Q}\left(x_{2}, z_{2}\right)$ in the image can be determined by analysing the interferogram formed from two images. It can be written as

$$
\delta \phi=\frac{4 \pi}{\lambda}\left(\rho^{\prime}-\rho\right)=\frac{4 \pi}{\lambda} \Delta \rho,
$$

where

$$
\begin{aligned}
\Delta \rho= & \Delta x\left[\frac{x_{2}}{d_{2}}+\frac{h x_{2} z_{2}}{d_{2}^{3}}\right]-\Delta z\left[\frac{h-z_{2}}{d_{2}}-\frac{h^{2} z_{2}}{d_{2}^{3}}\right] \\
& -\Delta x\left[\frac{x_{1}}{d_{1}}+\frac{h x_{1} z_{1}}{d_{1}^{3}}\right]+\Delta z\left[\frac{h-z_{1}}{d_{1}}+\frac{d^{2} z_{1}}{d_{1}^{3}}\right] .
\end{aligned}
$$

Hence, (14) can be written as

$$
\begin{aligned}
\Delta \phi= & \frac{4 \pi}{\lambda}\left[\Delta x\left(\frac{x_{2}}{d_{2}}-\frac{x_{1}}{d_{1}}\right)+\Delta z h\left(\frac{1}{d_{1}}-\frac{1}{d_{2}}\right)\right] \\
& +\frac{4 \pi}{\lambda}\left[\Delta x h\left(\frac{x_{2} z_{2}}{d_{2}^{3}}-\frac{x_{1} z_{1}}{d_{1}^{3}}\right)+\Delta z\left(\frac{z_{2}}{d_{2}}-\frac{z_{1}}{d_{1}}\right)+\Delta z h^{2}\left(\frac{z_{1}}{d_{1}^{3}}-\frac{z_{2}}{d_{2}^{3}}\right)\right] .
\end{aligned}
$$


The first term of the right hand side of (15) is independent of $z_{1}$ and $z_{2}$, which is the phase difference associated with the flat earth surface and can be termed as $\delta \phi_{0}$, while the second term, dependent on $z_{1}$ and $z_{2}$, contributes to the terrain elevation and is termed as $\delta \phi^{\prime}$.

Therefore, $\delta \phi$ can be written as

$$
\delta \phi=\delta \phi_{0}+\delta \phi^{\prime}
$$

For a flat earth area in the image, $\delta \phi^{\prime}=0$. Selecting two consecutive fringe points in the flat earth interferogram, $\delta \phi_{0}=2 \pi$. In this case, (15) reduces to

$$
\Delta x\left(\frac{x_{2}}{d_{2}}-\frac{x_{1}}{d_{1}}\right)+\Delta z h\left(\frac{1}{d_{1}}-\frac{1}{d_{2}}\right)=\frac{\lambda}{2} .
$$

$\Delta x$ and $\Delta z$ can be evaluated, assuming under one condition that $\Delta z$ is zero and under the second that $\Delta x=0$.

Case $i$ : When $\Delta z=0,(17)$ reduces to

$$
\Delta x=\frac{\lambda d_{1} d_{2}}{2\left(x_{2} d_{1}-x_{1} d_{2}\right)} \text {. }
$$

This is the same expression derived by Lin et al (1991). In this case, vertical shift of the satellite is assumed to be zero.

Case ii: When $\Delta x=0,(17)$ can be reduced as

$$
\Delta z=\frac{\lambda d_{1} d_{2}}{2 h\left(d_{2}-d_{1}\right)} \text {. }
$$

Hence, with prior knowledge of the type of repeat orbit of the satellite, the precise value of the horizontal or vertical baseline vector can be evaluated using the distance between two consecutive fringes of the phase interferogram of the flat earth. However, in reality, satellite shift is usually two-dimensional with both $\Delta x$ and $\Delta z$ components. To calculate $\Delta x$ and $\Delta z$, three consecutive fringe points of the phase interferogram of the flat earth can be used to develop mathematical formulation. For this $Q$ and $R$ points (figure 1) can be selected as fringe points for the flat earth where $\delta \phi_{0}=2 \pi$ and in analogy with (17), the following equation can be derived as

$$
\Delta x\left[\frac{x_{3}}{d_{3}}-\frac{x_{2}}{d_{2}}\right]+h \Delta z\left[\frac{1}{d_{2}}-\frac{1}{d_{3}}\right]=\frac{\lambda}{2} .
$$

Now, (17) and (20) can be written in the form

$$
\begin{aligned}
& \Delta x \mathrm{~A}_{1}+\Delta z \mathrm{~B}_{1}=c, \\
& \Delta x \mathrm{~A}_{2}+\Delta z \mathrm{~B}_{2}=c,
\end{aligned}
$$

where,

$$
\begin{aligned}
\mathrm{A}_{1} & =\frac{x_{2}}{d_{2}}-\frac{x_{1}}{d_{1}}, \quad \mathrm{~A}_{2}=\frac{x_{3}}{d_{3}}-\frac{x_{2}}{d_{2}}, \\
B_{1} & =h\left(\frac{1}{d_{1}}-\frac{1}{d_{2}}\right), \quad \mathrm{B}_{2}=h\left(\frac{1}{d_{2}}-\frac{1}{d_{3}}\right), \\
c & =\frac{\lambda}{2} .
\end{aligned}
$$




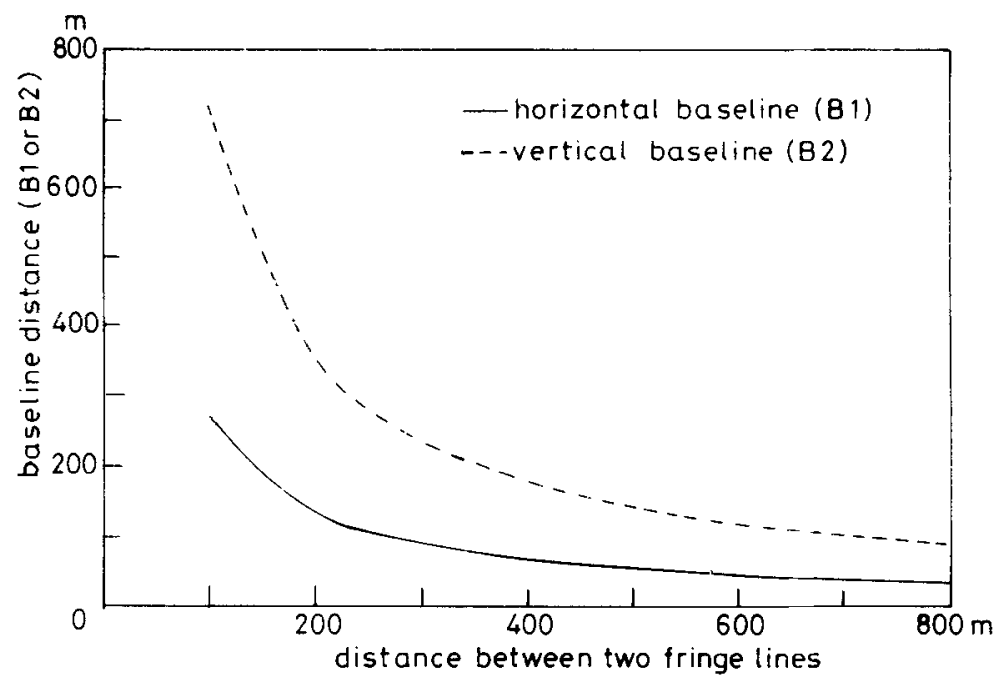

Figure 2. Variation of baseline with distance between fringe lines.

Equation (21) can be solved for $\Delta x$ and $\Delta z$ and can be written as:

$$
\begin{gathered}
\Delta x=\frac{c\left(\mathrm{~B}_{2}-\mathrm{B}_{1}\right)}{\mathrm{A}_{1} \mathrm{~B}_{2}-\mathrm{A}_{2} \mathrm{~B}_{1}}, \\
\Delta z=\frac{c\left(\mathrm{~A}_{2}-A_{1}\right)}{\mathrm{B}_{1} \mathrm{~A}_{2}-\mathrm{B}_{2} \mathrm{~A}_{1}} .
\end{gathered}
$$

By substituting the value of coefficients and simplifying (22) and (23), it can be shown that

$$
\begin{aligned}
& \Delta x=\frac{\lambda}{2}\left\{\frac{2 d_{1} d_{3}-d_{1} d_{2}-d_{2} d_{3}}{x_{1}\left(d_{2}-d_{3}\right)+x_{2}\left(d_{3}-d_{1}\right)+x_{3}\left(d_{1}-d_{2}\right)}\right\}, \\
& \Delta z=\frac{\lambda}{2 h}\left\{\frac{x_{1} d_{2} d_{3}-2 x_{2} d_{1} d_{3}+x_{3} d_{1} d_{2}}{x_{1}\left(d_{3}-d_{2}\right)+x_{2}\left(d_{1}-d_{3}\right)+x_{3}\left(d_{2}-d_{1}\right)}\right\} .
\end{aligned}
$$

Equations (24) and (25) give estimates of the two-dimensional orbital shifts ( $\Delta x$ and $\Delta z$ ) for SAR interferometry with the knowledge of three consecutive fringe points in the interferogram of the flat earth.

\section{Results and discussions}

Figure 2 explains the variation of estimated horizontal or vertical baseline with the increase of the distance between two consecutive fringe lines of the phase interferogram for $5.3 \mathrm{GHz}$ INSAR (ERS-1 C-band SAR) at a distance of 300 kilometres from the nadir $\left(x_{1}\right)$ using (18) and (19). It is observed that with increase of the distance between fringe lines of the flat earth, horizontal or vertical distance of the satellite in the repeat orbit decreases. The baseline vector also depends on the frequency of operation of the INSAR. It means that by knowing the fringe-line distance, either vertical shift or horizontal shift of the satellite 


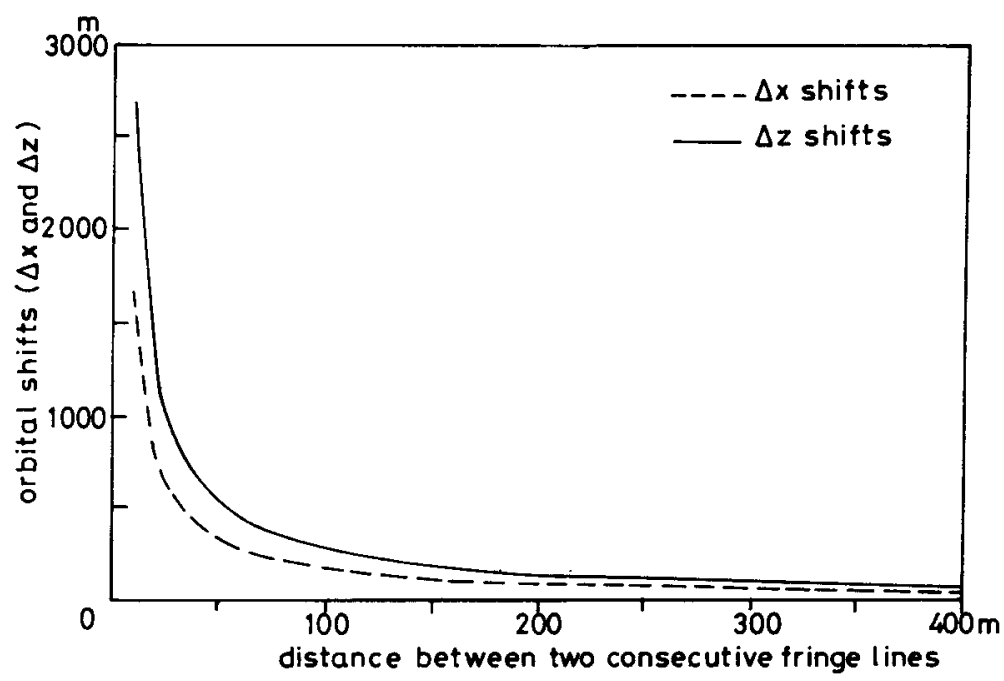

Figure 3. Variation of orbital shift for ERS C-band INSAR $\left(h=790 \mathrm{~km}, x_{1}=300 \mathrm{~km}\right)$.

repeat orbit can be estimated precisely and this used to develop the topographic map of the earth.

Figures 3 and 4 depict variation of orbital shift ( $\Delta x$ and $\Delta z$ ) with distance between two consecutive fringe lines for C-band ERS INSAR at distances from the nadir point of $300 \mathrm{~km}$ and $400 \mathrm{~km}$ respectively. It is observed that $\Delta x$ and $\Delta z$ orbital shifts decrease very fast with increase of distance between two consecutive fringe lines $(\Delta F L)$ up to $150 \mathrm{~m}$. Beyond this $\Delta \mathrm{FL}$, orbital shifts decrease slowly. It is also observed from both figures that vertical orbital shift $(\Delta z)$ and horizontal orbital shift $(\Delta x)$ are different for $x_{1}=300 \mathrm{~km}$ and $x_{1}=400 \mathrm{~km}$ at any value of $\Delta \mathrm{FL}$. In this discussion, equal spacing of fringe lines is assumed i.e., $\Delta \mathrm{FL}=\Delta \mathrm{FL}_{1}=\Delta \mathrm{FL}_{2}$, where $\Delta \mathrm{FL}_{1}$ is the distance between the first two fringe points and $\Delta \mathrm{FL}_{2}$ is the distance between the second and third fringe points.

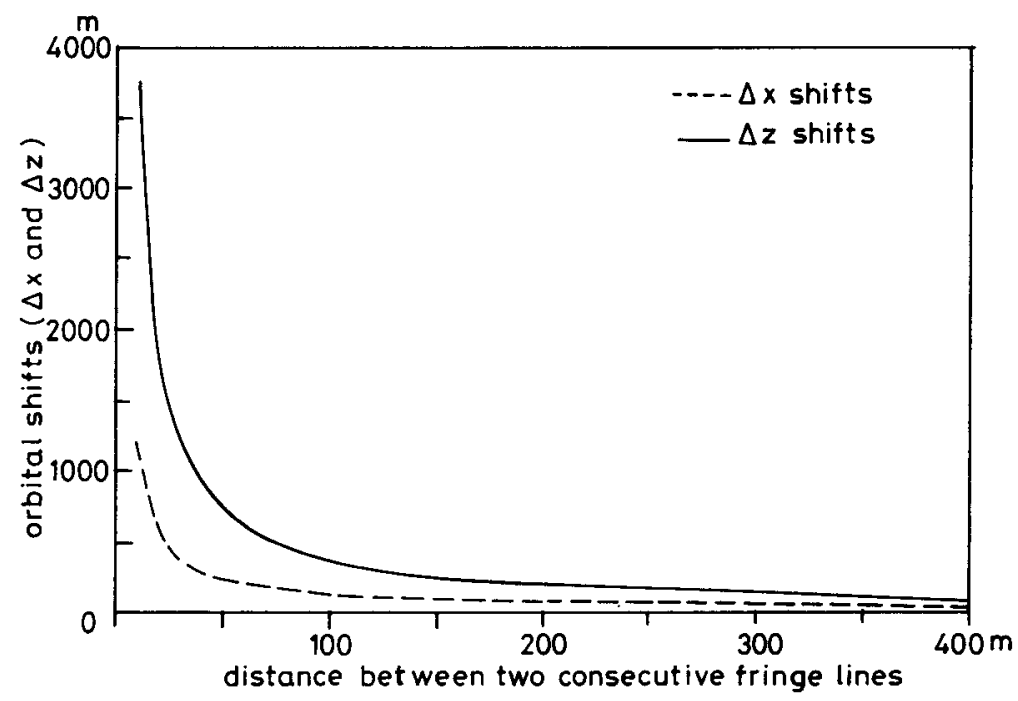

Figure 4. Variation of orbital shift for ERS C-band INSAR $\left(h=790 \mathrm{~km}, x_{1}=400 \mathrm{~km}\right)$. 


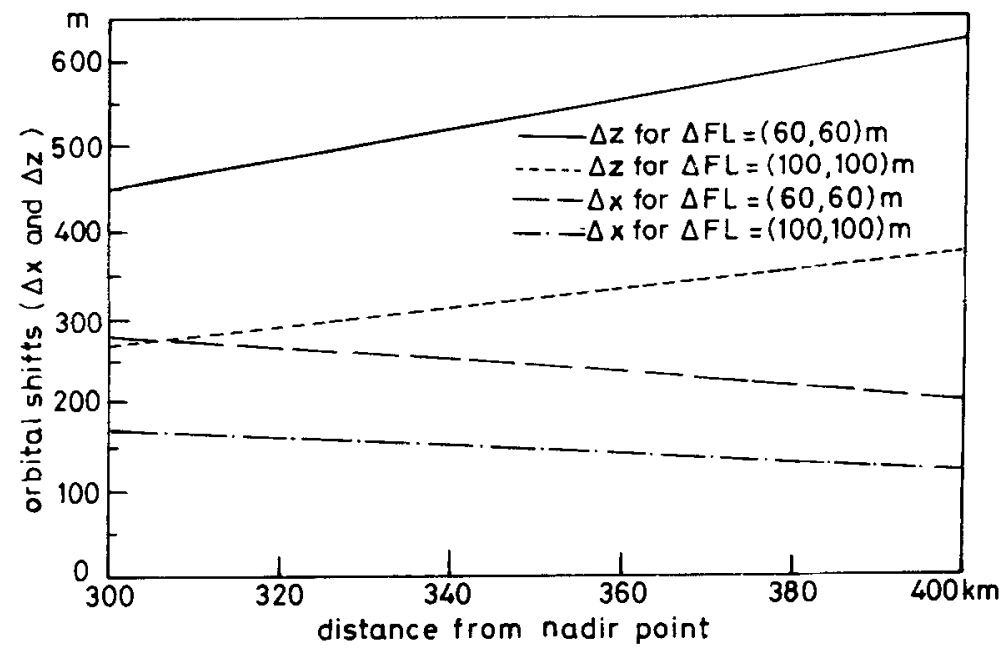

Figure 5. Variation of orbital shifts with distance from nadir point for three equally spaced consecutive fringe lines.

Figure 5 illustrates the variation of orbital shift $\Delta x$ and $\Delta z$ with distance from the nadir point $\left(x_{1}\right)$ for $\Delta F L=60 \mathrm{~m}$ and $\Delta F L=100 \mathrm{~m}$ of the interferogram of the flat earth surface of C-band ERS INSAR. It is observed that the horizontal shift decreases and the vertical shift increases with distance from the nadir point for both $\Delta \mathrm{FL} . \Delta x$ and $\Delta z$ variations for a particular $\Delta \mathrm{FL}$ form a divergence pattern as distance from the nadir point increases. Divergence characteristics of the variation of $\Delta x$ and $\Delta z$ orbital shifts decrease with increase of $\Delta F L$ values for the interferogram of flat earth surface.

Figure 6 explains the variation of the orbital shifts $(\Delta x$ and $\Delta z$ ) with relative change in $\left(\Delta F L_{1}, \Delta F L_{2}\right)$ for a point at a distance of $300 \mathrm{~km}$ from the nadir of the interferogram of flat earth surface. It is found that orbital shifts are very sensitive to the values of $\Delta \mathrm{FL}_{1}$

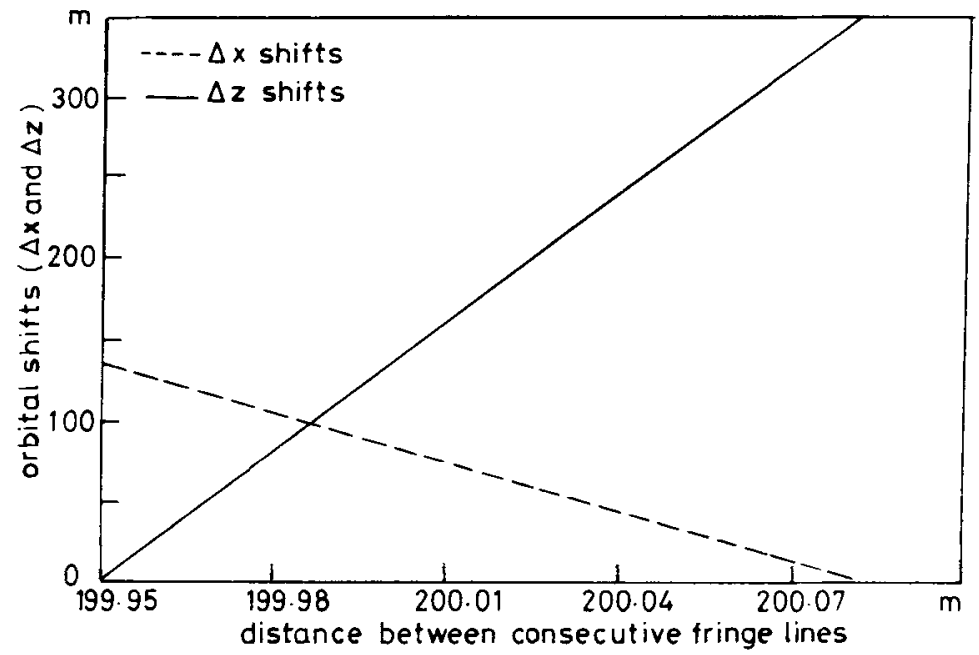

Figure 6. Effect on orbital orientation due to variation in the distance between three consecutive fringe lines. 


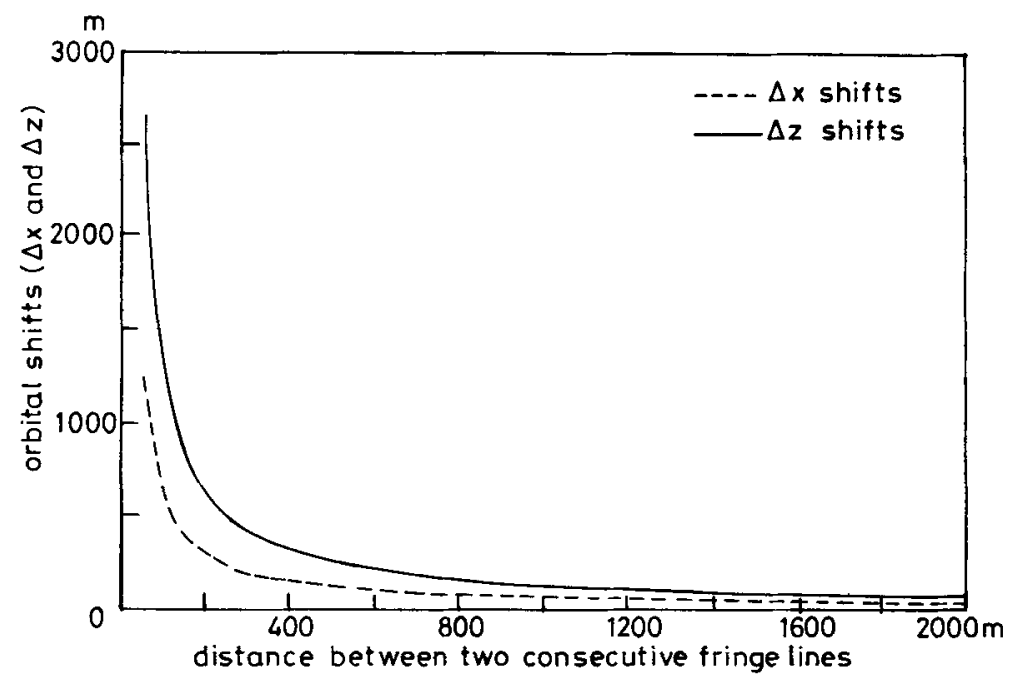

Figure 7. Variation of orbital shifts for SEASAT INSAR $\left(x_{1}=350 \mathrm{~km}\right)$.

and $\Delta \mathrm{FL}_{2}$. In this figure, it is assumed that the distance between the second and third consecutive fringe lines $\left(\Delta \mathrm{FL}_{2}\right)$ is $200 \mathrm{~m}$ and the distance between the first and second fringe line $\left(\Delta F L_{1}\right)$ varies from 199.95 to $200.1 \mathrm{~m}$. It is found that $\Delta x$ decreases and $\Delta z$ increases very fast with slow increase of $\Delta \mathrm{FL}_{1}$ while keeping $\Delta \mathrm{FL}_{2}$ constant. Hence, the resultant orbital vector $\left[\Delta \mathrm{FL}=\left(\Delta \mathrm{FL}_{1}^{2}+\Delta \mathrm{FL}_{2}^{2}\right)^{1 / 2}\right]$ increases with increase in the value of $\Delta \mathrm{FL}_{1}$ or the angle of the resultant vector with the horizontal vector. Therefore the exact knowledge of position of three consecutive fringe lines in the interferogram of the flat earth surface is essential for prediction of precise satelite-orbital shift in repeat pass INSAR.

Figure 7 explains the variation of orbital shift with increase in the distance between two fringe lines $(\triangle \mathrm{FL})$ for L-band SEASAT SAR at a distance $x_{1}=350 \mathrm{~km}$. It is observed

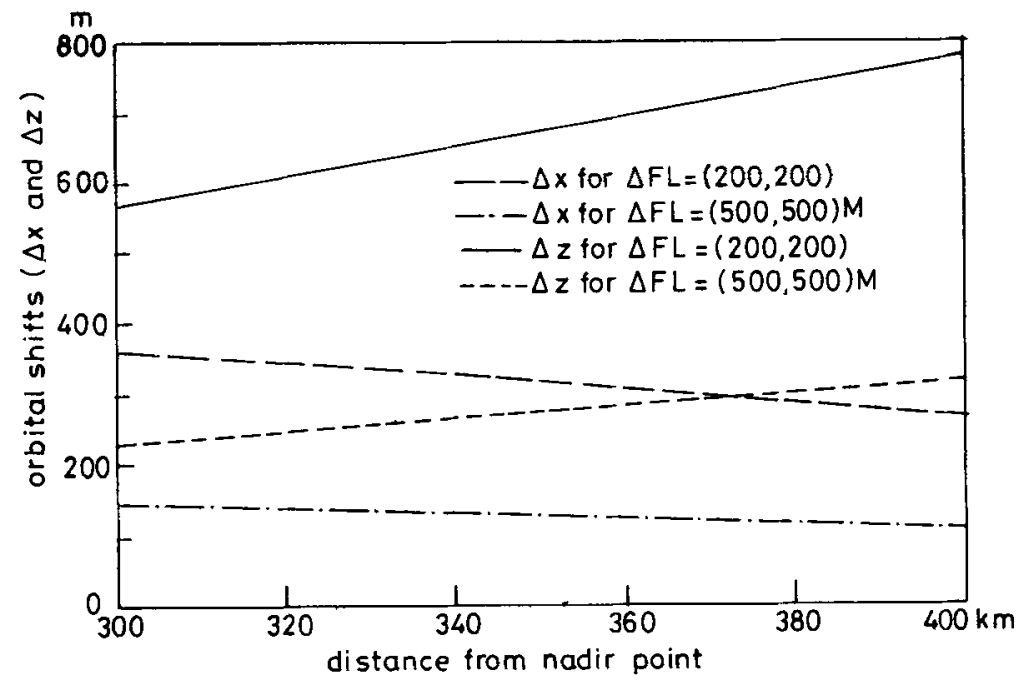

Figure 8. Variation of orbital shifts with distance from nadir point for three equally spaced consecutive fringe lines. 


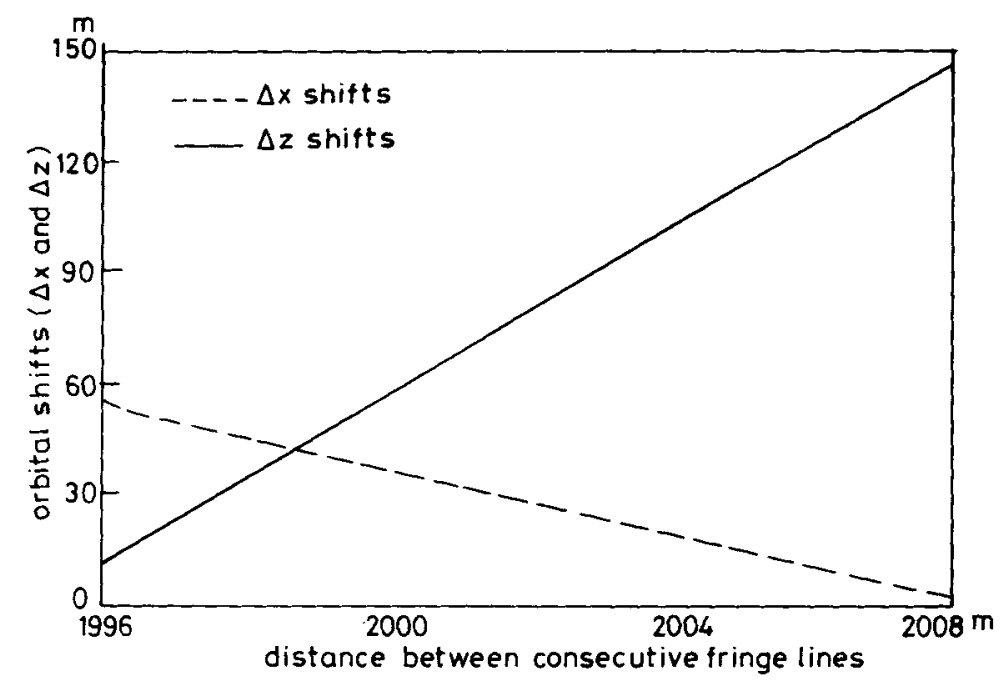

Figure 9. Effect on orbital orientation due to variation in the distance between three consecutive fringe lines.

that $\Delta x$ and $\Delta z$ decrease rapidly up to $\Delta F L=(400 \mathrm{~m}, 400 \mathrm{~m})$ and beyond this value, decrease slowly. Orbital shift depends upon $\Delta \mathrm{FL}, x_{1}$ and frequency. It is also found from figure 4 that for the same value of orbital shift, the required distance between consecutive fringe lines $(\triangle F L)$ is less for C-band INSAR as compared to L-band SEASAT INSAR.

Figure 8 illustrates the variation of orbital shift with $x_{1}$ for $\triangle F L=(200 \mathrm{~m}, 200 \mathrm{~m})$ and $\Delta F L=(500 \mathrm{~m}, 500 \mathrm{~m})$ at L-band SEASAT INSAR. In this the vertical shift increases and the horizontal shift decreases with increase of fringe point distance from the nadir $\left(x_{1}\right)$. It is also observed that the slope of increase or decrease of $\Delta z$ and $\Delta x$ decreases with the increase of $\Delta F L$ from $(200 \mathrm{~m}, 200 \mathrm{~m})$ to $(500 \mathrm{~m}, 500 \mathrm{~m})$. In general, at any point of fringe location $\left(x_{1}\right)$, orbital shift is higher for $\Delta F L=(200 \mathrm{~m}, 200 \mathrm{~m})$ compared to $\Delta F L(500 \mathrm{~m}$, $500 \mathrm{~m}$ ).

Figure 9 depicts the effect of $\left(\Delta F L_{1}, \Delta F L_{2}\right)$ on orbital shifts in the interferogram of plane earth of SEASAT INSAR. In this case, $\triangle \mathrm{FL}_{2}(2000 \mathrm{~m})$ is taken to be constant and $\Delta F L_{1}$ varies in relation to $\Delta \mathrm{FL}_{2}$ from $1996 \mathrm{~m}$ to $2008 \mathrm{~m}$. It is observed that due to little change in the value of $\Delta \mathrm{FL}_{1}$ compared to $\mathrm{FL}_{2}$, orbital shifts ( $\Delta x$ and $\Delta z$ ) change drastically in orientation. It is found that $\Delta x$ decreases and $\Delta z$ increases with change in $\Delta \mathrm{FL}_{1}$ relative to $\Delta \mathrm{FL}_{2}$. Hence, knowledge of the exact distance between three consecutive fringe lines of the interferogram of flat earth is essential to obtain the precise value of $\Delta x$ and $\Delta z$. Hence, the resultant orbital vector increases with increase in the value of $\Delta F L_{1}$ as compared to that of $\Delta \mathrm{FL}_{2}$.

\section{Conclusion}

In this paper mathematical formulations have been developed for evaluation of onedimensional (horizontal or vertical) and two-dimensional orbital shifts of repeat pass INSAR based on the knowledge of two or three consecutive fringe lines in the interferogram of flat earth. The precise knowledge of orbital shifts ( $\Delta x$ or $\Delta z$ ) or ( $\Delta x$ and $\Delta z)$ 
is required for evaluation of terrain mapping through INSAR. It is found that knowing the distance between three consecutive fringe lines is very useful for obtaining the exact orientation of the orbital shift, which depends upon frequency, distance of the fringe-line location from the nadir point and distance between two fringe lines $(\Delta F L)$. Orbital orientation plays an important role in the mathematical formulation used for evaluation of terrain elevation map using repeat pass INSAR which will be different for one- or two-dimensional orbital shifts.

The authors are grateful to Shri V P Sandlas, for constant encouragement and fruitful discussions. Thanks are also due to Dr K K Jha for critical comments and review.

\section{References}

Hagberg J O, Ulander L M H 1993 On the optimization of Interferometric SAR for topographic mapping. IEEE Trans. Geosci. Remote Sensing 31: 303-307

Li F K, Goldstein R M 1990 Studies of multibaseline spaceborne interferometric synthetic aperture radar. IEEE Trans. Geosci. Remote Sensing 28: 88-97

Lin Q, Vesecky J F, Zebker H A 1991 Topography estimation with interferometric synthetic aperture radar using fringe detection. Proc. Int. Geosci. Remote Sensing Symp. pp 2173-2176

Lin Q, Vesecky J F, Zebker H A 1992 New approaches in the interferometric SAR data processing. IEEE Trans. Geosci. Remote Sensing 30: 560-567

Madsen S N, Zebker H A, Martin J 1993 Topographic mapping using radar interferometry: processing technique. IEEE Trans. Geosci. Remote Sensing 31: 246-256

Zebker H A, Goldstein R M 1986 Topographic mapping from interferometric synthetic aperture radar observations. J. Geophys. Res. 91: 4993-4999 